ROCZNIKI FILOZOFICZNE

Tom LXVIII, numer $4-2020$

DOI: https://doi.org/10.18290/rf20684-16

TOMASZ JARMUŻEK

\& ALEKSANDER PAROL

\title{
ON SOME LANGUAGE EXTENSION OF LOGIC MR: A SEMANTIC AND TABLEAU APPROACH
}

\section{INTRODUCTION}

Positional logic is a family of logical systems in which among the logical constants we can distinguish positional operator. $\mathcal{R}$-operator is a propositional operator which connects two arguments: $\alpha$ which denotes an entity belonging to the semantic category of names and $A$ which denotes a sentence.

In Ajdukiewicz's categorization it is symbolized as $\frac{s}{n, s}$.

Propositions constructed with it, of the form $\mathcal{R}_{\alpha}(A)$, are read as 'the sentence $A$ is realized at condition $\alpha$ '. It was pointed out, for example in Tkaczyk (2009), that positional operator could be interpret as a specific kind of conjunction which connects the variables of two categories and the truth value of the sentence is based on this specific connection.

The history of $\mathcal{R}$-operator is strictly related to the history of temporal logic as first systems of temporal logic were, in fact, positional systems of logic with $\mathcal{R}$-operator interpreted as temporal realization. Therefore the propositions of the form $\mathcal{R}_{\alpha}(A)$ were read as 'sentence $A$ is realized at the time $\alpha$ '. The first system of this kind and the first system of temporal logic at all, was constructed by Jerzy Łoś in his master thesis (Łoś 1947). ${ }^{1}$

Tomasz JARMUŻeK, PhD Habil., Associate Professor at the Nicolaus Copernicus University in Torun (UMK), Faculty of Philosophy and Social Sciences, Department of Logic; address for correspondence: ul. Stanisława Moniuszki 16/20, 87-100 Toruń; e-mail: jarmuzek@ umk.pl; ORCID: https:// orcid.org/ 0000-0003-3456-3859),

AleKsander PAROL, Bachelor of Arts, Cardinal Stefan Wyszynski University in Warsaw, Institute of Philosophy; e-mail: alekparo192@gmail.com; ORCID: https://orcid.org/0000-00017700-1800.

${ }^{1}$ More about examples of temporal applications can be found in JARMUŻEK 2006; 2013; 2018, 
Łoś's positional calculus was created to fulfill the objective of formal analysis of John Stuart Mill's Canons of Induction. This was achieved by noticing that the relation between the cause and the effect should not be considered in a timeless conditions in which this relations could be modeled by the simple connective of conjunction. Embedding the temporal aspect of the cause-effect relation was done by using positional operator. In Łoś's work this operator was symbolized by uppercase letter "U" (MALINOWSKI, Pietrowicz, and SZALACHA-JaRMUŻEK 2020).

Some proposition of full formal reconstruction of the Loś's system was given in Jarmużek ad Tkaczyk (2019). We can say that the underlying logic is first order logic with quantification ranging over various types of variables. To this fundamental system, we should add U-operator and functional constant $\delta$, which can form expressions of the form $\mathrm{U}_{\alpha}(A)$ and $\mathrm{U}_{\delta\left(\alpha_{1}, \epsilon_{2}\right)}(A)$, where $\alpha, \alpha_{1}, \epsilon_{2}, \delta\left(\alpha_{1}, \epsilon_{2}\right)$ belong to the set of terms ( $\epsilon_{1}$ denotes an interval and $\delta\left(\alpha_{1}, \epsilon_{2}\right)$ denotes the position that occurs in time after interval $\epsilon_{2}$ passed from $\alpha_{1}$ ) and $A$ belongs to the set of propositions made of propositional variables. Therefore, the set of terms is expanded with the expressions of the form $\delta\left(\alpha_{1}, \epsilon_{2}\right)$ and the set of formulas by the expressions of the form $\mathbf{U}_{\alpha}(A)$ and $\mathrm{U}_{\delta\left(\alpha_{1}, \epsilon_{2}\right)}(A)$ using logical connectives and quantifiers ranging over all kinds of variables, but without nesting of $U$. All expressions which are of the form of a tautology of first order logic, are axioms of the Loś calculus. Additionally the axiomatic system is expanded by the six axioms referring to the usage of the symbols $\mathrm{U}$ and $\delta$.

The positional logic was created and published by Łoś in 1947 in Poland. But until 1951 when Henryk Hiż wrote a review of his paper in English (Hiż 1951) results of Łoś were not widely known (TKACZYK 2009). This two-paged review printed in Journal of Symbolic Logic, caused rich expansion in the field of positional logics in the $50 \mathrm{~s}$ and the $60 \mathrm{~s}$. It is worth to mention here the works of Arthur N. Prior, Peter Geach, James Garson and Nicholas Rescher, which were crucial for development of Łoś idea (TKACZYK 2009, 32-34). The last of them proposed as a first, usage of $P$ instead of $U$, and finally $\mathcal{R}$ which is widely use until the present day.

Rescher's systems of propositional logic was mostly based on the system of Łoś. But on the other hand they were simpler - quantifying was limited

but particularly position RESCHER URQUHART 1971 is recommendable. It should be noted that also the first system of epistemic logic was made through the application of realization operator, and also by Jerzy Łoś. But this result was only partially recognized on the Western side of Iron Curtain thanks to the review of Roman Suszko (1949); see also: LeChniaK 1988. 
and was not allowed to range over propositional variables nor over other expressions. The system of Topological Logic was the most general of them and as its author showed in the article by Garson and Rescher $(1968,8)$. Aforementioned system can be used to formulate preceding systems of chronological logics, this name Rescher uses for less general systems of positional logic, interpreted temporally.

The most recent and simplest of the positional logic is the system of MR created by Jarmużek and Pietruszczak (2004). MR is the simplest form of positional logic as it forbids nesting of $\mathcal{R}$-operator, it is based on Classical Propositional Logic (in short: $\mathrm{CPL}$ ) without quantification, the set of axioms is much smaller than of other mentioned systems. ${ }^{2}$ From the other hand, its simplicity allows to extend it according to the demands and desired interpretation of the $\mathcal{R}$-operator. MR could be extended and applied to the specific philosophical problems. We intend to show it in the present. A very wide spectrum of such extensions has been recently suggested by Jarmużek and Tkaczyk (2019). Although these extensions are motivated by applications to reasoning about social phenomena, we will examine two of them and show also non-sociological applications.

\section{IDEA OF THE PAPER}

In this section we will present system MR and point at some possibility of its extension proposed in Malinowski, Pietrowicz, and Szalacha-Jarmużek (2020). To introduce the alphabet of MR, we need distinguish sets of symbols:

- logical connectives $\operatorname{Con}=\{\neg, \wedge, \vee, \rightarrow, \leftrightarrow\}$,

- variables $\operatorname{Var}=\left\{p_{i}: i \in \mathbb{N}\right\}$,

- positional letters $\mathrm{PL}=\left\{a_{i}: i \in \mathbb{N}\right\}$,

- brackets: ),(,

- realization operator $\mathcal{R}$.

Having the definition of alphabet, we can define the set of auxiliary expressions and the set of formulas.

Definition 2.1 (Auxiliary Expressions) The set of auxiliary expressions $A E_{M R}$ is the smallest set satisfying conditions stated below:

\footnotetext{
${ }^{2}$ For more about some interesting metalogical properties of MR see: JARMUŻEK and TKACZYK 2015; KARCZEWSKA 2018.
} 
- $\operatorname{Var} \subseteq A E_{M R}$,

- $\neg A \in A E_{M R}$, for any $A \in A E_{M R}$,

- $A_{1} \xi A_{2} \in A E_{M R}$, for any $A_{1}, A_{2} \in A E_{M R}$ and $\xi \in \operatorname{Con} \backslash\{\neg\}$.

Let us notice that $A E_{M R}$ is just the set of all formulas of CPL.

Definition 2.2 (Formulas MR) The set of MR formulas For ${ }_{M R}$ is the smallest set satisfying conditions stated below:

1. $\mathcal{R}_{\alpha}(A) \in$ For $_{M R}$, for any $A \in A E_{M R}$ and $\alpha \in P L$,

2. $\neg \phi \in$ For $_{M R}$, for any $\phi \in$ For $_{M R}$,

3. $\phi_{1} \xi \phi_{2} \in$ For $_{M R}$, for any $\phi_{1}, \phi_{2} \in$ For $_{M R}$ and $\xi \in$ Con $\backslash\{\neg\}$.

Let us assume that $A \in \mathrm{AE}_{\mathrm{MR}}$, we assume that $\vDash_{\mathrm{CPL}} A$ means that $A$ is a tautology of CPL. In turn by Sub we mean a uniform substitution of formulas $A E_{M R}$ with For $_{M R}$. The only rule of inference used in Jarmużek and Pietruszczak (2004) for the axiomatization of MR is detachment rule:

$$
\frac{\phi_{1}, \phi_{1} \rightarrow \phi_{2}}{\phi_{2}}
$$

The authors assume also four axioms:

$$
\begin{aligned}
& \operatorname{Sub}(A), \text { where } \vDash_{\mathrm{CPL}} A \\
& \mathcal{R}_{\alpha}(\neg A) \leftrightarrow \neg \mathcal{R}_{\alpha}(A) \\
& \mathcal{R}_{\alpha}\left(A_{1}\right) \wedge \mathcal{R}_{\alpha}\left(A_{2}\right) \rightarrow \mathcal{R}_{\alpha}\left(A_{1} \wedge A_{2}\right) \\
& \mathcal{R}_{\alpha}(A), \text { where } \vDash_{C P L} A
\end{aligned}
$$

The logic syntactically defined by (MP) and the four given axioms is just MR. In the standard way, by this axiomatic machinery and the notion of proof, we determine syntactical relation $\vdash_{\text {MR }}$. Now, let us present the semantics.

Definition 2.3 (Model for MR) A model $\mathfrak{M}$ for the set For $_{M R}$ is any triple $\langle W, d, v\rangle$, where:

- Wis a non-empty set of positions

- $d: P L \rightarrow W$ is a denotation of positional letters

- $v: W \times A E_{M R} \rightarrow\{0,1\}$ is such a valuation of $A E_{M R}$ in positions that for all $w \in W$ and $A_{1}, A_{2}$ : 
1. $\boldsymbol{v}\left(w, \neg A_{1}\right)=1$ iff $\boldsymbol{v}\left(w, A_{1}\right)=0$,

2. $\boldsymbol{v}\left(w, A_{1} \wedge A_{2}\right)=1$ iff $\boldsymbol{v}\left(w, A_{1}\right)=1$ and $\boldsymbol{v}\left(w, A_{2}\right)=1$,

3. $v\left(w, A_{1} \vee A_{2}\right)=1$ iff $v\left(w, A_{1}\right)=1$ or $v\left(w, A_{2}\right)=1$,

4. $\boldsymbol{v}\left(w, A_{1} \rightarrow A_{2}\right)=1$ iff $\boldsymbol{v}\left(w, A_{1}\right)=0$ or $\boldsymbol{v}\left(w, A_{2}\right)=1$,

5. $v\left(w, A_{1} \leftrightarrow A_{2}\right)=1$ iff $\boldsymbol{v}\left(w, A_{1}\right)=\boldsymbol{v}\left(w, A_{2}\right)$.

Basing on the previous definitions we can present the notion of truth in a model.

Definition 2.4 (Truth in model MR) Let $\mathfrak{M}=\langle W, d, v\rangle$ be a model for MR. Formula $\phi$ is true in $\mathfrak{M}$ (in short: $\mathfrak{M} \vDash \phi$ ) iff it satisfies one of the conditions:

1. $\phi=\mathcal{R}_{\alpha}(A)$ and $v(d(\alpha), A)=1$, for some $\alpha \in P L$ and $A \in A E_{M R}$;

2. for all $\phi_{1}, \phi_{1} \in$ For $_{M R}$ :

(a) if $\phi=\neg \phi_{1}$ then it is not the case that $\mathfrak{M} \vDash \phi_{1}$ (in short: $\mathfrak{M} \not \models \phi_{1}$ ),

(b) if $\phi=\phi_{1} \wedge \phi_{2}$ then $\mathfrak{M} \vDash \phi_{1}$ and $\mathfrak{M} \vDash \phi_{2}$,

(c) if $\phi=\phi_{1} \vee \phi_{2}$ then $\mathfrak{M} \vDash \phi_{1}$ or $\mathfrak{M} \vDash \phi_{2}$,

(d) if $\phi=\phi_{1} \rightarrow \phi_{2}$ then $\mathfrak{M} \not \models \phi_{1}$ or $\mathfrak{M} \vDash \phi_{2}$,

(e) if $\phi=\phi_{1} \leftrightarrow \phi_{2}$ then either $\mathfrak{M} \vDash \phi_{1}$ and $\mathfrak{M} \vDash \phi_{2}$ or $\mathfrak{M} \not \models \phi_{1}$ and

$\mathfrak{M} \not \models \phi_{2}$.

The restrictions regarding interpretation of symbols concern positional letters and variables. In the first case the nature of positions is not determined by the interpretation, nor by the properties of the mathematical structures which are used as formal semantics for the system. Also, no restrictions are provided for the denotations of elements of the set Var. By default, MR models determine semantic relation $\vDash_{\text {MR }}$

As the authors proved in Jarmużek and Pietruszczak (2004), MR if an axiomatic system is sound and complete with respect to the presented class of models, so: $\vdash_{\mathrm{MR}}=\vdash_{\mathrm{MR}}$. MR is the smallest normal positional logic, simple and general in the interpretation. ${ }^{3}$ Those factors are big advantages, as MR could be used as a foundation for a more complex calculus.

\footnotetext{
${ }^{3}$ In book Jarmużek and Tkaczyk (2015) the criterion of being a normal positional logic is defined as preserving Boolean interpretation of all classical connectives in scope of $\mathcal{R}$-operator. It is worth to mention that also weaker (non-normal) systems of positional logic exists (see: TKACZYK 2013; 2018; JARMUŻEK 2007).
} 
Extending the language of system of MR could lead to fruitful investigations on applications of positional logic to the philosophical problems. As Rescher and Garson (1968) wrote, applications of positional logic are very wide - from the first intended usage, that is to model temporal statements in physics, through the modal interpretation, to the meta-mathematical interpretation of realization operator. In this paper we want to extend the language of MR by:

1. allowing $\mathcal{R}$-operator to range over not only positional letters but also sequences of positional letters (like in the example: $\mathcal{R}_{a_{1}, \ldots, a_{n}}(p)$ )

2. adding expressions built with $n$-ary predicate symbols and positional letters.

This could lead to strengthening the expression power of the MR and therefore lead to possible new domains of applications. Both extensions have been mentioned and the first even proposed in Malinowski, Pietrowicz, and Szalacha-Jarmużek (1920) to adapt positional logic to the needs of reasoning about a social phenomena.

A good example for 2. comes from Set Theory. Let $p_{1}$ denote a sentence 'set is finite', positional letters $a_{1}, a_{2}$ denote sets, and let $P$ denote the relation of $\subset$. Then we can form the true sentence in the extended language:

$$
\mathcal{R}_{a_{2}}\left(p_{1}\right) \wedge P\left(a_{1}, a_{2}\right) \rightarrow \mathcal{R}_{a_{1}}\left(p_{1}\right)
$$

Here we are stating that if the sentence $p_{1}$ is realized at the position $a_{2}$, and the set denoted by position $a_{2}$ is such set that the set denoted by the $a_{1}$ is a subset of it, then the sentence $p_{1}$ is realized also at the position $a_{1}$ as $p_{1}$ denotes "set is finite." In turn, some example of applications of 1 . we will propose in the section 6 .

\section{LANGUAGE AND SEMANTICS OF MRE}

Basing on the minimal system for $\mathcal{R}$-operator, we would like to extend the expressive power of the logic. When we think about using $\mathcal{R}$-operator for modelling some sentences, it comes out that taking into account just one position, in which the sentence is realized is shallowing the interpretation. As we know that description of the phenomena of which we do not have a full knowledge, should allow us to add some factors and relations between them as our knowledge progresses (see Malinowski, Pietrowicz, and SzALACHA-JARMUŻEK 2020). We would like to model sentences more accurately. 
The aforementioned way of extending MR needs an previous extension of the alphabet of the original system by adding a set of $n$-ary predicate symbols. Therefore, to the alphabet of MR we add:

- predicates PS $=\left\{P_{n}^{i}: i, n \in \mathbb{N}\right\}$,

where $i$ is arity of a given predicate symbol.

Firstly, we will define the set of auxiliary expressions, and then, the set of formulas of the presented system which we will denote as MRE .

The set $A E_{M R E}$ of auxiliary expressions is, in fact, equal to the set of all formulas of $C P L$, defined in definition 2.1. In turn set For ${ }_{M R E}$ we obtain by replacing in definition of formulas For $_{\text {MRE }} 2.2$ condition 1. with the following two conditions:

$$
\begin{aligned}
& \mathcal{R}_{\alpha_{1}, \ldots, \alpha_{i}}(A) \in \text { For }_{\mathrm{MRE}} \text {, for any } A \in A E_{M R E} \text { and } \alpha_{1}, \ldots, \alpha_{i} \in \mathrm{PL} \\
& \vartheta\left(\alpha_{1}, \ldots, \alpha_{i}\right) \in \text { For }_{\mathrm{MRE}} \text {, for any } i, n \in \mathbb{N}, \vartheta_{n}^{i} \in \mathrm{PS} \text {, and } \alpha_{1}, \ldots, \alpha_{i} \in \mathrm{PL} .
\end{aligned}
$$

Comparing definition of For $_{\text {MRE }}$ to 2.2 , we can notice that our logic is extended in a intended way. We can create a formula which contains more than one symbol of a position and among the formulas of MRE we can find also the expressions containing predicates containing positional letters as arguments. Moreover, For ${ }_{\text {MRE }} \subset$ For $_{\text {MRE }}$.

Having the formal definition of the MRE formulas, we will extend the semantics, created in Jarmużek and Pietruszczak (2004) for MR. We need some auxiliary notions. For any $n \in \mathbb{N}$, let us denote n-ary Cartesian product $\underbrace{X \times, \ldots, \times X}_{n}$ of a set $X$ as $X^{n}$. If $n=1$, then $X^{n}=X$. In case of ordered n-tuple $\left\langle x_{1}, \ldots, x_{n}\right\rangle \in X^{n}$, we will write $\left(x_{1}, \ldots, x_{n}\right)$ or just $x_{1}, \ldots, x_{n}$, without brackets. Additionally, the power set of a set $X$ we will denote by $P(X)$, while the union of all sets contained in $X$, as $\bigcup X$.

Since in the language we have additional formulas with predicate symbols, a model for MRE is not like before, a triple, but a quadruple, containing an additional function for the denotation of predicates.

Definition 3.1 (Model for MRE) A model $\mathfrak{M}$ for the set For $r_{M R E}$ is any quadruple $\langle W, d, \tilde{d}, v\rangle$, where:

- $W$ is a non-empty set of positions

- $d: P L \rightarrow W$ is a denotation of positional letters 
- $\tilde{d}: P S \rightarrow \bigcup\left\{P\left(W^{i}\right): i \in \mathbb{N}\right\}$, where for any $\vartheta_{n}^{i} \in P S, \tilde{d}\left(\vartheta_{n}^{i}\right) \subseteq W^{i}$, is a denotation of predicates.

- $v: \bigcup\left\{W^{i}: i \in \mathbb{N}\right\} \times A E_{\text {MRE }} \rightarrow\{0,1\}$ is such a valuation of $A E_{M R}$ in an ordered n-tuples of positions that for all $n \in \mathbb{N},\left(w_{1}, \ldots, w_{n}\right) \in$ $\bigcup\left\{W^{i}: i \in \mathbb{N}\right\}$, and $A_{1}, A_{2}$ :

1. $v\left(\left(w_{1}, \ldots, w_{n}\right), \neg A_{1}\right)=1$ iff $v\left(\left(w_{1}, \ldots, w_{n}\right), A_{1}\right)=0$,

2. $v\left(\left(w_{1}, \ldots, w_{n}\right), A_{1} \wedge A_{2}\right)=1$ iff $v\left(\left(w_{1}, \ldots, w_{n}\right), A_{1}\right)=1$ and

$$
v\left(\left(w_{1}, \ldots, w_{n}\right), A_{1}\right)=1\left(\left(w_{1}, \ldots, w_{n}\right), A_{2}\right)=1,
$$

3. $v\left(\left(w_{1}, \ldots, w_{n}\right), A_{1} \vee A_{2}\right)=1$ iff $v\left(\left(w_{1}, \ldots, w_{n}\right), A_{1}\right)=1$ or $v\left(\left(w_{1}, \ldots, w_{n}\right), A_{2}\right)=1$,

4. $v\left(\left(w_{1}, \ldots, w_{n}\right), A_{1} \rightarrow A_{2}\right)=1$ iff $v\left(\left(w_{1}, \ldots, w_{n}\right), A_{1}\right)=0$ or

$$
v\left(\left(w_{1}, \ldots, w_{n}\right), A_{2}\right)=1,
$$

5. $\boldsymbol{v}\left(\left(w_{1}, \ldots, w_{n}\right), A_{1} \leftrightarrow A_{2}\right)=1$ iff $\boldsymbol{v}\left(\left(w_{1}, \ldots, w_{n}\right), A_{1}\right)=v\left(\left(w_{1}, \ldots, w_{n}\right), A_{2}\right)$.

Now, we define the notion of truth in a model of MRE. We just modify the analogous definition for MR 2.4. We generalize point 1. in 2.4 and add a condition for predicate expressions:

Definition 3.2 (Truth in model of MRE) Let $\mathfrak{M}=\langle W, d, \tilde{d}, v\rangle$ be a model for MR. Formula $\phi$ is true in $\mathfrak{M}$ (in short: $\mathfrak{M} \vDash \phi$ ) iff it satisfies one of the conditions:

1. $\phi=\mathcal{R}_{\alpha_{1}, \ldots, \alpha_{n}}(A)$ and $v\left(\left(d\left(\alpha_{1}\right), \ldots, d\left(\alpha_{n}\right), A\right)=1\right.$, for some $n \in \mathbb{N}$, $\alpha_{1}, \ldots, \alpha_{n} \in P L$, and $A \in A E_{M R}$;

2. $\phi=\vartheta_{n}^{i}\left(\alpha_{1}, \ldots, \alpha_{i}\right)$ and $\left(d\left(\alpha_{1}\right), \ldots, d\left(\alpha_{i}\right)\right) \in \tilde{d}\left(\vartheta_{n}^{i}\right)$, for some $\vartheta_{n}^{i} \in P S$, $\alpha_{1}, \ldots, \alpha_{i} \in P L$

3. for all $\phi_{1}, \phi_{1} \in$ For $_{\text {MRE }}$ :

(a) if $\phi=\neg \phi_{1}$ then it is not the case that $\mathfrak{M} \vDash \phi_{1}$ (in short: $\mathfrak{M} \not \models \phi_{1}$ ),

(b) if $\phi=\phi_{1} \wedge \phi_{2}$ then $\mathfrak{M} \vDash \phi_{1}$ and $\mathfrak{M} \vDash \phi_{2}$,

(c) if $\phi=\phi_{1} \vee \phi_{2}$ then $\mathfrak{M} \vDash \phi_{1}$ or $\mathfrak{M} \vDash \phi_{2}$,

(d) if $\phi=\phi_{1} \rightarrow \phi_{2}$ then $\mathfrak{M} \not \models \phi_{1}$ or $\mathfrak{M} \vDash \phi_{2}$,

(e) if $\phi=\phi_{1} \leftrightarrow \phi_{2}$ then either $\mathfrak{M} \vDash \phi_{1}$ and $\mathfrak{M} \vDash \phi_{2}$ or $\mathfrak{M} \not \models \phi_{1}$ and $\mathfrak{M} \not \models \phi_{2}$. 
Now, in the standard way we define relation $\vDash_{\text {MRE }}$ :

Definition 3.3 (MRE Semantic Consequence Relation) Let $\Phi \cup\{\phi\} \subseteq$ For $_{M R}$. Formula $\phi$ follows from a set of formulas $\Phi$ in MRE ( $\left.\Phi \vDash_{\text {MRE }} \phi\right)$ iff for every model $\mathfrak{M}$ for MRE, if $\mathfrak{M} \vDash \Phi$ then $\mathfrak{M} \vDash \phi$.

\section{TABLEAUX FOR MRE}

Having defined the semantic form of MRE, we will construct a proof theory. Our proposal here are tableaux methods. To achieve this result, we will extend the tableaux method introduced for MR in Jarmużek and Tkaczyk $(2015,128-131){ }^{4}$

For tableaux proofs we assume that the set of tableau expressions $A E_{t}$ is the smallest set that contains all expressions of the form:

$(\Gamma, A)$,

where $\Gamma$ is an ordered tuple $\left(\alpha_{1}, \ldots, \alpha_{i}\right), \alpha_{1}, \ldots, \alpha_{i} \in \mathrm{PL}$, and $A \in \mathrm{AE}_{\mathrm{MRE}}$. Therefore $\mathrm{AE}_{t}=\bigcup\left\{P L^{i}: i \in \mathbb{N}\right\} \times \mathrm{AE}_{\mathrm{MRE}}$. When it does not lead to confusion, we will omit brackets, writing $\Gamma, A$ instead of $(\Gamma, A)$.

The set of tableau expression in which we carry tableaux proofs is the set $\mathrm{Ex}_{t}=\mathrm{For}_{\mathrm{MRE}} \cup A \mathrm{E}_{t}$. Now we introduce the notion of tableau inconsistency, so the notion of a set we look for when decomposing expressions in a tableau proof.

Definition 4.1 (Tableau Inconsistency) Let $\Sigma \subseteq E x_{t} . \quad \Sigma$ is tableau inconsistent iff at least one of the following conditions is satisfied:

- there is $A \in A E_{\text {MRE }}$, such that $A, \neg A \in \Sigma$,

- there is such a pair $(\Gamma, A) \in A E_{t}$, that $(\Gamma, A),(\Gamma, \neg A) \in \Sigma$.

A set of tableau expressions is tableau consistent iff it is not tableau consistent.

Having this, let us present the set of tableaux rules, which we will be using. These rules are divided into five mutually exclusive sets of rules and this division seems to be self-explanatory.

\footnotetext{
${ }^{4}$ It is worth to underline that in Jarmużek and Tkaczyk (2015): (i) two kinds of tableaux for MR were introduced - here we decide to develop the version presented on p. 130; (ii) no proofs that both kinds are complete with respect to the semantics for MR were delivered.
} 
Positive rules for classical connectives:
$(\wedge) \frac{A_{1} \wedge A_{2}}{A_{1}, A_{2}}$
( $) \frac{A_{1} \vee A_{2}}{A_{1} \mid A_{2}}$
$(\rightarrow) \frac{A_{1} \rightarrow A_{2}}{\neg A_{1} \mid A_{2}}$
$(\leftrightarrow) \frac{A_{1} \leftrightarrow A_{2}}{A_{1}, A_{2} \mid}$

Negative rules for classical connectives:

$$
\begin{array}{cc}
(\neg \neg) \frac{\neg \neg A}{A} & (\neg \wedge) \frac{\neg\left(A_{1} \wedge A_{2}\right)}{\neg A_{1} \mid \neg A_{2}} \\
(\neg \vee) \frac{\neg\left(A_{1} \vee A_{2}\right)}{\neg A_{1}, \neg A_{2}} & (\neg \rightarrow) \frac{\neg\left(A_{1} \rightarrow A_{2}\right)}{\neg A_{1}, A_{2}} \\
(\neg \leftrightarrow) \frac{\neg\left(A_{1} \leftrightarrow A_{2}\right)}{A_{1}, \neg A_{2} \mid \neg A_{1}, A_{2}}
\end{array}
$$

\section{Elimination rules:}
$(\mathcal{R}) \frac{\mathcal{R}_{\Gamma}(A)}{\Gamma, A}$
$(\neg \mathcal{R}) \frac{\neg \mathcal{R}_{\Gamma}(A)}{\Gamma, \neg A}$

Positive rules for auxiliary expressions:

$$
\begin{array}{lc}
(\Gamma, \wedge) \frac{\Gamma,\left(A_{1} \wedge A_{2}\right)}{\Gamma, A_{1}, \Gamma, A_{2}} & (\Gamma, \vee) \frac{\Gamma,\left(A_{1} \vee A_{2}\right)}{\Gamma, A_{1} \mid \Gamma, A_{2}} \\
(\Gamma, \rightarrow) \frac{\Gamma,\left(A_{1} \rightarrow A_{2}\right)}{\Gamma, \neg A_{1} \mid \Gamma, A_{2}} & (\Gamma, \leftrightarrow) \frac{\Gamma,\left(A_{1} \leftrightarrow A_{2}\right)}{\Gamma, \neg A_{1}, \Gamma \neg A_{2} \mid \Gamma, A_{1}, \Gamma, A_{2}}
\end{array}
$$

Negative rules for auxiliary expressions:

$$
\begin{array}{cr}
(\Gamma, \neg \neg) \frac{\Gamma \neg \neg A}{\Gamma, A} & (\Gamma, \neg \wedge) \frac{\Gamma, \neg\left(A_{1} \wedge A_{2}\right)}{\Gamma, \neg A_{1} \mid \Gamma, \neg A_{2}} \\
(\Gamma, \neg \vee) \frac{\Gamma, \neg\left(A_{1} \vee A_{2}\right)}{\Gamma, \neg A_{1}, \Gamma, \neg A_{2}} & (\Gamma, \neg \rightarrow) \frac{\Gamma, \neg\left(A_{1} \rightarrow A_{2}\right)}{\Gamma, A_{1}, \neg \Gamma, A_{2}} \\
(\Gamma, \neg \leftrightarrow) \frac{\Gamma, \neg\left(A_{1} \leftrightarrow A_{2}\right)}{\Gamma, A_{1}, \Gamma, \neg A_{2} \mid \Gamma, \neg A_{1}, \Gamma, A_{2}}
\end{array}
$$

Let TR denote the set of all the rules listed. For any rules from TR expressions in the numerator will be called input, while expressions from the denominator will be called output. Let us take as an example the rule $(\Gamma, \neg \neg)$. 
One of its inputs is $\left(a_{1}, \neg \neg p\right)$ and then the corresponding output is $\left(a_{1}, p\right)$. Notice that this rule is a non-branching one, i.e. for any input, it has got only one output. On the other hand, $(\neg \wedge)$ is a branching rule and in this case we have two outputs, for example: $\neg \mathcal{R}_{a_{2}}(q)$ and $\neg \mathcal{R}_{a_{2}, a_{4}}(p \wedge r)$, if the input is $\neg\left(\mathcal{R}_{a_{2}}(q) \wedge \mathcal{R}_{a_{2}, a_{4}}(p \wedge r)\right)$. Let us sometimes treat inputs and outputs of these rules as sets. Once we have the notions of input and output we can define the notion of applicability of a rule. ${ }^{5}$

Definition 4.2 (Applicability) Let $(r) \in T R$ and $\Sigma \in E x_{t}$, we say that $(r)$ is applicable to $\Sigma$ iff an input of $(r)$ is a subset of $\Sigma$.

We define the tableau consequence relation by referring to the concept of closure under tableau rules.

Definition 4.3 (Tableau Closure) Let $X \subseteq T R$ and $\Sigma, \Phi \subseteq E x_{t} . \Phi$ is a closure of $\Sigma$ under tableau rules from $X$ iff there exists such a subset of natural numbers $K$ that:

- $K=\mathbb{N}$ or $K=\{1,2,3, \ldots, n\}$ for some $n \in \mathbb{N}$

- there exists such an injective function $f: K \rightarrow\left\{Y: Y \subseteq E x_{t}\right\}$ that:

$-Y_{1}=\Sigma$

- for all $i, i+1 \in K$ there exists such tableau rule $(r) \in X$ that its input is included in $Y_{i}$ and one of its corresponding outputs is equal to $Y_{i+1} \backslash Y_{i}$

- for all $i, i+1 \in K$, for any tableau rule $(r) \in X$, if ( $r$ )'s input is included in $Y_{i}$ and one of $(r)$ 's corresponding outputs is equal to $Y_{i+1} \backslash Y_{i}$, then there are no such $j, j+1 \in K$ that $j>i$ and one of the remaining outputs of $(r)$ is equal to $Y_{j+1} \backslash Y_{j}$

- for any $(r) \in T R$ if ( $r$ )'s input is included in $\bigcup_{i \in K} Y_{i}$, then one of the corresponding outputs of $(r)$ is in $\bigcup_{i \in K} Y_{i}$

- $\Phi=\bigcup_{i \in K} Y_{i}$.

\footnotetext{
${ }^{5}$ The tableau metatheoretical notions and facts we present are partially based on article JARMUŻEK and KLONOWSKI 2020. Here we adapted them to the context of positional logic.
} 
Definition 4.4 (MRE Tableau Proof) Let $\Sigma \cup\{A\} \subseteq$ For $_{\text {MRE. }}$ A is a tableau consequence of $\Sigma$ in MRE ( $\Sigma \triangleright_{T R} A$ ) iff there is a finite set $\Phi \subseteq \Sigma$ such that any closure under tableau rules from $T R$ of $\Phi \cup\{\neg A\}$ is tableau inconsistent.

Therefore, defining tableau consequence, we can say that thesis for the presented tableau system is any expression that is a tableau consequence of the empty set.

Definition 4.5 (Suitability) Let $\mathfrak{M}=\langle W, d, \tilde{d}, v\rangle$ be a model for MR and $\Sigma \subset E x_{t} \cdot \mathfrak{M}$ is suitable for $\Sigma$ iff for any $A \in F_{\text {For }}{ }_{M R E}$ and any $\alpha_{1}, \ldots, \alpha_{n} \in P L$ :

- if $A \in \Sigma$, then $\mathfrak{M} \vDash_{\mathrm{MRE}} A$;

- if $\left(\left(\alpha_{1}, \ldots, \alpha_{n}\right), A\right) \in \Sigma$ then $v\left(\left(d\left(\alpha_{1}\right), \ldots, d\left(\alpha_{n}\right)\right), A\right)=1$.

Now, we will present two lemmas that we need for the proof of Soundness Theorem.

Lemma 4.6 Let $\Sigma \in E x_{t}$ and $\mathfrak{M}=\langle W, d, \tilde{d}, v\rangle$ be a model for MRE. Let $\mathfrak{M}$ be suitable for $\Sigma$. For any $(r) \in T R$, if $(r)$ has been applied to $\Sigma$, then $\mathfrak{M}$ is suitable for the union of $\Sigma$ and at least one of the output of $(r)$.

Proof. Assume all the hypotheses. For the cases in which the rules for external classical connectives are applied, the proof is obvious.

- Suppose that $(\mathcal{R})$ has been applied to the $\Sigma$. Then according to definition 4.2, $\mathcal{R}_{\alpha_{1}, \ldots, \alpha_{n}}(A) \in \Sigma$, for some $\alpha_{1}, \ldots, \alpha_{n} \in \mathrm{PL}$ and $A \in$ For $_{\text {MRE }}$, and we have got output $\left(\left(\alpha_{1}, \ldots, \alpha_{n}\right), A\right)$. Because model $\mathfrak{M}$ is suitable for $\Sigma$, by definition $3.2, \quad \mathfrak{M} \vDash \mathcal{R}_{\alpha_{1}, \ldots, \alpha_{\eta}}(A)$. Therefore $\mathrm{v}\left(\left(d\left(\alpha_{1}\right), \ldots, \mathrm{d}\left(\alpha_{n}\right)\right), A\right)=1$. By definition $4.5, \mathfrak{M}$ is suitable for $\Sigma \cup\left\{\left(\left(\alpha_{1}, \ldots, \alpha_{n}\right), A\right)\right\}$.

- Suppose that $(\neg \mathcal{R})$ has been applied to the $\Sigma$. Then according to definition $4.2, \neg \mathcal{R}_{\alpha_{1}, \ldots, \alpha_{n}}(A) \in \Sigma$, for some $\alpha_{1}, \ldots, \alpha_{n} \in \mathrm{PL}$ and $A \in$ For $_{\text {MRE }}$, and we have got output $\left(\left(\alpha_{1}, \ldots, \alpha_{n}\right), \neg A\right)$. Because model $\mathfrak{M}$ is suitable for $\Sigma$, by definition 3.2, $\mathfrak{M} \not \models \mathcal{R}_{\alpha_{1}, \ldots, \alpha_{n}}(A)$. Therefore $\mathrm{v}\left(\left(d\left(\alpha_{1}\right), \ldots, \mathrm{d}\left(\alpha_{n}\right)\right), A\right)=0$, and $v\left(\left(d\left(\alpha_{1}\right), \ldots, d\left(\alpha_{n}\right)\right), \neg A\right) \stackrel{\alpha_{1} \ldots, \alpha_{n}}{=}$. By definition 4.5, $\mathfrak{M}$ is suitable for $\Sigma \cup\left\{\left(\left(\alpha_{1}, \ldots, \alpha_{n}\right), \neg A\right)\right\}$.

- Suppose that $(\Gamma, \vee)$ has been applied to the $\Sigma$. Then according to definition 4.2, $\left(\left(\alpha_{1}, \ldots, \alpha_{n}\right), A_{1} \vee A_{2}\right) \in \Sigma$, for some $\alpha_{1}, \ldots, \alpha_{n} \in \mathrm{PL}$ and $A_{1}, A_{2} \in$ For $_{\mathrm{MRE}}$, and we have got output $\left(\left(\alpha_{1}, \ldots, \alpha_{n}\right), A_{1}\right)$ or $\left(\left(\alpha_{1}, \ldots, \alpha_{n}\right), A_{2}\right)$. Because model $\mathfrak{M}$ is suitable for, by definition 4.5 . So, by definition 3.1 , or . Therefore, by definition 4.5, is suitable for or for. 
- Suppose that $(\Gamma, \neg \neg)$ has been applied to the $\sum$. Then according to definition 4.2, $\left(\left(\alpha_{1}, \ldots, \alpha_{n}\right), \neg \neg A\right) \in \Sigma, \quad$ for some $\alpha_{1}, \ldots, \alpha_{n} \in \mathrm{PL}$ and $\neg \neg A) \in$ For $_{\text {MRE }}$, and we have got output $\left(\left(\alpha_{1}, \ldots, \alpha_{n}\right), A\right)$. Because model $\mathfrak{M}$ is suitable for $\Sigma$, by definition $4.5, \mathrm{v}\left(\left(d\left(\alpha_{1}\right), \ldots, \mathrm{d}\left(\alpha_{n}\right)\right), \neg \neg A\right)=1$. So, by definition 3.1, $\mathrm{v}\left(\left(d\left(\alpha_{1}\right), \ldots, \mathrm{d}\left(\alpha_{n}\right)\right), A\right)=1$. Therefore, by definition $4.5, \mathfrak{M}$ is suitable for $\Sigma \cup\left(\left(\alpha_{1}, \ldots, \alpha_{n}\right), A\right)$.

- For the remaining rules, the proofs are analogous.

Theorem 4.7 (Soundess Theorem) Let $\Sigma \cup\{A\} \subseteq$ For $_{\text {MRE }}$. Then $\Sigma \triangleright_{T R} A \Rightarrow$ $\Sigma \vDash_{\text {MRE }} A$.

Proof. Let $\Sigma \cup\{A\} \subseteq$ For $_{\text {MRE }}$. Suppose by transposition that $\Sigma \not \nvdash_{\text {MRE }} A$. So there is a model $\mathfrak{M}$ for MRE such that $\mathfrak{M} \vDash \Sigma$ and $\mathfrak{M} \vDash \neg A$. By definition 4.5, $\mathfrak{M}$ is suitable for $\Sigma \cup\{\neg A\}$. It means that for any finite $\Sigma^{\prime} \subseteq \Sigma, \mathfrak{M}$ is suitable for $\Sigma^{\prime} \cup\{\neg A\}$. By lemma 4.6 for any such $\Sigma^{\prime}$ there exists at least one closure under $T R$ of $\Sigma^{\prime} \cup\{\neg A\}$ that is tableau consistent. So, by 4.4, it is not the case that $\Sigma \triangleright_{T R} A$.

Next we will introduce the notion of a model generated by a tableau consistent closure under $T R$.

Definition 4.8 ( $\Sigma$-model) Let $\Sigma \subseteq E x_{t}$ be a tableau consistent closure under TR. A model generated by $\Sigma$ (for short $\Sigma$-model) is a model $\left(W_{\Sigma}, d_{\Sigma}, \tilde{d}_{\Sigma}, v_{\Sigma}\right)$ such that:

- $y \in W_{\Sigma}$ iff there are $\alpha_{1}, \ldots, \alpha_{n} \in P L$, such that for some $1 \leq i \leq n$, $y=\alpha_{i}$ and:

- there is $p_{i} \in$ Var such that $\left(\left(\alpha_{1}, \ldots, \alpha_{n}\right), p_{i}\right) \in \Sigma$, or

- there is $P_{n}^{i} \in P S$ such that $P_{n}^{i}\left(\alpha_{1}, \ldots, \alpha_{n}\right) \in \Sigma$,

- denotation of positional letters $d_{\Sigma}: P L \rightarrow W_{\Sigma}$ satisfies conditions:

1. $d_{\Sigma}\left(\alpha_{i}\right)=\alpha_{i}$, if $\alpha_{i} \in W_{\Sigma}$

2. $d_{\Sigma}\left(\alpha_{i}\right)=\alpha_{j}$, where $j$ is the smallest number among indexes of those positional letters that belong to $W_{\Sigma}$, if $\alpha_{i} \notin W_{\Sigma}$,

- denotation of predicate symbols $\tilde{d}_{\Sigma}: P S \rightarrow \bigcup\left\{P\left(W^{i}\right): i \in \mathbb{N}\right\}$ satisfies condition: $\left(d_{\Sigma}\left(\alpha_{1}\right), \ldots, d_{\Sigma}\left(\alpha_{i}\right)\right) \in \tilde{d}_{\Sigma}\left(\vartheta_{n}^{i}\right)$ iff $\vartheta_{n}^{i}\left(\alpha_{1}, \ldots, \alpha_{i}\right) \in \Sigma$, 
- $\quad v: \bigcup\left\{W^{i}: i \in \mathbb{N}\right\} \times A E_{M R E} \rightarrow\{0,1\}$ is such a valuation of $A E_{M R}$ in ordered $n$-tuples of positions that for all $n \in \mathbb{N}$,

$\left(\alpha_{1}, \ldots, \alpha_{n}\right) \in \bigcup\left\{W^{i}: i \in \mathbb{N}\right\}$, all $p_{i} \in$ Var , and all $A_{1}, A_{2} \in A E_{\text {MRE }}$.

1. $\left.v\left(\left(\alpha_{1}, \ldots, \alpha_{n}\right), p_{i}\right)\right)=1$ iff $\left(\left(\alpha_{1}, \ldots, \alpha_{i}\right), p_{j}\right) \in \Sigma$,

2. $v\left(\left(\alpha_{1}, \ldots, \alpha_{n}\right), \neg A_{1}\right)=1$ iff $v\left(\left(\alpha_{1}, \ldots, \alpha_{n}\right), A_{1}\right)=0$,

$$
v\left(\left(\alpha_{1}, \ldots, \alpha_{n}\right), A_{1} \wedge A_{2}\right)=1 \text { iff } v\left(\left(\alpha_{1}, \ldots, \alpha_{n}\right), A_{1}\right)=1 \text { and }
$$$$
v\left(\left(\alpha_{1}, \ldots, \alpha_{n}\right), A_{2}\right)=1 \text {, }
$$

3. $v\left(\left(\alpha_{1}, \ldots, \alpha_{n}\right), A_{1} \vee A_{2}\right)=1$ iff $v\left(\left(\alpha_{1}, \ldots, \alpha_{n}\right), A_{1}\right)=1$ or

$$
v\left(\left(\alpha_{1}, \ldots, \alpha_{n}\right), A_{2}\right)=1
$$

4. $v\left(\left(\alpha_{1}, \ldots, \alpha_{n}\right), A_{1} \rightarrow A_{2}\right)=1$ iff $v\left(\left(\alpha_{1}, \ldots, \alpha_{n}\right), A_{1}\right)=0$ or

$$
v\left(\left(\alpha_{1}, \ldots, \alpha_{n}\right), A_{2}\right)=1
$$

5. $v\left(\left(\alpha_{1}, \ldots, \alpha_{n}\right), A_{1} \leftrightarrow A_{2}\right)=1$ iff $v\left(\left(\alpha_{1}, \ldots, \alpha_{n}\right), A_{1}\right)=v\left(\left(\alpha_{1}, \ldots, \alpha_{n}\right), A_{2}\right)$.

Before the next lemma, let us note that the complexity of the formula $A \in \mathrm{Ex}_{\mathrm{t}}$ we define in the standard way with no extension for $\mathcal{R}$-operator, so as the function which assigns to a formula a natural number with a respect to the number of occurrences of only classical logical connectives. Let $c(A)$ represent the result of application of complexity function to expression $A$.

Lemma 4.9 Let $\Sigma$ be a tableau consistent closure under TR. Let $\mathfrak{M}=\left\langle W_{\Sigma}, d_{\Sigma}, \tilde{d}_{\Sigma}, v_{\Sigma}\right\rangle$ be the model generated by $\Sigma$. If $\left(\left(\alpha_{1}, \ldots, \alpha_{n}\right), A\right) \in \Sigma$, then $v_{\Sigma}\left(\left(d_{\Sigma}\left(\alpha_{1}\right), \ldots, d_{\Sigma}\left(\alpha_{n}\right)\right), A\right)=1$, for any $\alpha_{1}, \ldots, \alpha_{n} \in P L$ and $A \in A E_{M R E}$.

Proof. Assume all the hypotheses. Let $\mathfrak{M}$ be a $\Sigma$-model such that $\mathfrak{M}=\left\langle W_{\Sigma}, d_{\Sigma}, \tilde{d}_{\Sigma}, v_{\Sigma}\right\rangle$, determined according to definition 4.8 .

Base case. Let us consider two atomic cases. $A=p_{i}$ or $A=\neg p_{i}$, where $p_{i} \in \operatorname{Var}$. If $\left.\left(\left(\alpha_{1}, \ldots \alpha_{n}\right)\right), p_{i}\right) \in \Sigma$, for some $\alpha_{1}, \ldots, \alpha_{n} \in \mathrm{PL}$, then, by construction of model 4.8: $v_{\Sigma}\left(\left(d_{\Sigma}\left(\alpha_{1}\right), \ldots, d_{\Sigma}\left(\alpha_{n}\right)\right), p_{i}\right)=1$. If $\left.\left(\left(\alpha_{1}, \ldots \alpha_{n}\right)\right), \neg p_{i}\right) \in \Sigma$, for some any $\alpha_{1}, \ldots, \alpha_{n} \in \mathrm{PL}$ and $p_{i} \in \mathrm{Var}$, then, by construction of a model 4.8: $\mathrm{v}_{\Sigma}\left(\left(\mathrm{d}_{\Sigma}\left(\alpha_{1}\right), \ldots, \mathrm{d}_{\Sigma}\left(\alpha_{n}\right)\right), \neg p_{i}\right)=1$. It is because $\mathrm{v}_{\Sigma}\left(\left(\mathrm{d}_{\Sigma}\left(\alpha_{1}\right), \ldots, \mathrm{d}_{\Sigma}\left(\alpha_{n}\right)\right), p_{i}\right)=0$, by construction of a model 4.8 , as $\left.\left(\left(\alpha_{1}, \ldots \alpha_{n}\right)\right), p_{i}\right) \notin \Sigma$, since $\Sigma$ is a tableau consistent closure under TR.

Inductive hypothesis. Let $n \in \mathbb{N}$. Suppose that for any $\alpha_{1}, \ldots, \alpha_{n} \in \mathrm{PL}$ and any $B \in \mathrm{AE}_{\mathrm{MRE}}$, such that $c(A) \leq n$, if $\left(\left(\alpha_{1}, \ldots, \alpha_{n}\right), B\right) \in \Sigma$, then $\mathrm{v}_{\Sigma}\left(\left(\mathrm{d}_{\Sigma}\left(\alpha_{1}\right), \ldots, \mathrm{d}_{\Sigma}\left(\alpha_{n}\right)\right), B\right)=1$. 
Inductive step. Let $c(A)=n+1$. We have nine cases to examine.

Let $A=C \vee D$ and $\left(\left(\alpha_{1}, \ldots \alpha_{n}\right), C \vee D\right) \in \Sigma$, for some $\alpha_{1}, \ldots, \alpha_{n} \in \mathrm{PL}$ and $C, D \in \mathrm{AE}_{\text {MRE }}$. Since $\Sigma$ is a tableau consistent closure under TR, by application of tableau rule $(\Gamma, \vee)$, also $\left(\left(\alpha_{1}, \ldots \alpha_{n}\right), C\right) \in \Sigma$ or $\left(\left(\alpha_{1}, \ldots \alpha_{n}\right), D\right) \in \Sigma$. However, by inductive hypothesis $\mathrm{v}_{\Sigma}\left(\left(\mathrm{d}_{\Sigma}\left(\alpha_{1}\right), \ldots, \mathrm{d}_{\Sigma}\left(\alpha_{n}\right)\right), C\right)=1$ or $v_{\Sigma}\left(\left(\mathrm{d}_{\Sigma}\left(\alpha_{1}\right), \ldots, \mathrm{d}_{\Sigma}\left(\alpha_{n}\right)\right), D\right)=1$. Therefore, by notion of a generated model 4.8, $\mathrm{v}_{\Sigma}\left(\left(\mathrm{d}_{\Sigma}\left(\alpha_{1}\right), \ldots, \mathrm{d}_{\Sigma}\left(\alpha_{n}\right)\right), C \vee D\right)=1$.

Let $A=\neg \neg C$ and $\left(\left(\alpha_{1}, \ldots \alpha_{n}\right), \neg \neg C\right) \in \Sigma$, for some $\alpha_{1}, \ldots, \alpha_{n} \in \mathrm{PL}$ and $C \in \mathrm{AE}_{\mathrm{MRE}}$. Since $\Sigma$ is a tableau consistent closure under TR, by application of tableau rule $(\Gamma, \neg \neg)$, also $\left(\left(\alpha_{1}, \ldots \alpha_{n}\right), C\right) \in \Sigma$. However, by inductive hypothesis $\mathrm{v}_{\Sigma}\left(\left(\mathrm{d}_{\Sigma}\left(\alpha_{1}\right), \ldots, \mathrm{d}_{\Sigma}\left(\alpha_{n}\right)\right), C\right)=1$. Therefore, by notion of a generated model 4.8, $\mathrm{v}_{\Sigma}\left(\left(\mathrm{d}_{\Sigma}\left(\alpha_{1}\right), \ldots, \mathrm{d}_{\Sigma}\left(\alpha_{n}\right)\right), \neg \neg C\right)=1$.

For the remaining cases, the proofs are analogous, since we have at disposal the corresponding tableau rules.

Now we can prove the analogous lemma for MRE formulas.

Lemma 4.10 Let $\Sigma$ be a tableau consistent closure under TR. Then there is a model $\mathfrak{M}=\langle W, d, \tilde{d}, v\rangle$ for $\mathbf{M R E}$ such that: if $\varphi \in \Sigma$, then $\mathfrak{M} \vDash \varphi$, for any $\varphi \in$ For $_{\text {MRE }}$.

Proof. Assume all the hypotheses. Let $\mathfrak{M}$ be a $\Sigma$-model such that $\mathfrak{M}=\left\langle W_{\Sigma}, d_{\Sigma}, \tilde{d}_{\Sigma}, v_{\Sigma}\right\rangle$, determined according to definition 4.8.

Base case. We have to consider two atomic cases.

Let $\varphi=P_{n}^{i}\left(\alpha_{1}, \ldots, \alpha_{i}\right)$, for some $P_{n}^{i} \in \mathrm{PS}$ and $\alpha_{1}, \ldots, \alpha_{i} \in \mathrm{PL}$. Then by definition of generated model 4.8, $\left(\mathrm{d}_{\Sigma}\left(\alpha_{1}\right), \ldots, \mathrm{d}_{\Sigma}\left(\alpha_{i}\right)\right) \in \mathrm{d}_{\Sigma}\left(P_{n}^{i}\right)$. Therefore $\mathfrak{M} \vDash P_{n}^{i}\left(\alpha_{1}, \ldots, \alpha_{i}\right)$.

Let $\varphi=\mathcal{R}_{\alpha_{1}, \ldots, \alpha_{i}}(A)$, for some $\alpha_{1}, \ldots, \alpha_{i} \in \mathrm{PL}$ and $A \in \mathrm{AE}_{\mathrm{MRE}}$. Since $\Sigma$ is a tableau consistent closure under $T R$, so by tableau rule $(\mathcal{R}),\left(\left(\alpha_{1}, \ldots, \alpha_{i}\right)\right.$, $A) \in \Sigma$. Consequently, by lemma $4.9, \mathbf{v}_{\Sigma}\left(\left(\mathrm{d}_{\Sigma}\left(\alpha_{1}\right), \ldots, \mathrm{d}_{\Sigma}\left(\alpha_{i}\right)\right), A\right)=1$, and by notion of truth in model 3.2, $\mathfrak{M} \vDash \mathcal{R}_{\alpha_{1}, \ldots, \alpha_{i}}(A)$.

Inductive hypothesis. Let $n \in \mathbb{N}$. Suppose that for any $\chi \in$ For $_{\text {MRE }}$ such that $c(\chi) \leq n$, if $\chi \in \Sigma$, then $\mathfrak{M} \vDash \chi$.

Inductive step. Let $c(A)=n+1$. We have eleven cases to examine.

Let $\varphi=\neg P_{n}^{i}\left(\alpha_{1}, \ldots, \alpha_{i}\right)$, for some $P_{n}^{i} \in \mathrm{PS}$ and $\alpha_{1}, \ldots, \alpha_{i} \in \mathrm{PL}$. Then by definition of a generated model $4.8,\left(\mathrm{~d}_{\Sigma}\left(\alpha_{1}\right), \ldots, \mathrm{d}_{\Sigma}\left(\alpha_{i}\right)\right) \notin \tilde{\mathrm{d}}_{\Sigma}\left(P_{n}^{i}\right)$, since $\Sigma$ is a tableau consistent closure under TR. Therefore, by notion of truth in model 3.2, $\mathfrak{M} \vDash \neg P_{n}^{i}\left(\alpha_{1}, \ldots, \alpha_{i}\right)$.

Let $\varphi=\neg \mathcal{R}_{\alpha_{1}, \ldots, \alpha_{i}}(A)$, for some $\alpha_{1}, \ldots, \alpha_{i} \in \mathrm{PL}$ and $A \in \mathrm{AE}_{\mathrm{MRE}}$. Since $\Sigma$ is a tableau consistent ${ }^{2}$ closure under TR, so by tableau rule $(\neg \mathcal{R}),\left(\left(\alpha_{1}, \ldots, \alpha_{i}\right)\right.$, 
$\neg A) \in \Sigma$. Consequently, by the lemma 4.9, $v_{\Sigma}\left(\left(\mathrm{d}_{\Sigma}\left(\alpha_{1}\right), \ldots, \mathrm{d}_{\Sigma}\left(\alpha_{i}\right)\right), \neg A\right)=1$, and by notion of model generated 4.8, $\mathrm{v}_{\Sigma}\left(\left(\mathrm{d}_{\Sigma}\left(\alpha_{1}\right), \ldots, \mathrm{d}_{\Sigma}\left(\alpha_{i}\right)\right), A\right)=0$. Hence by the notion of truth in model 3.2, $\mathfrak{M} \vDash \neg \mathcal{R}_{\alpha_{1}, \ldots, \alpha_{i}}(A)$.

Let $\varphi=\neg \neg \phi$, for some $\phi \in$ For $_{\text {MRE }}$. Since $\Sigma{ }^{i}$ is a tableau consistent closure under TR, by application of tableau rule $(\neg \neg)$, also $\phi \in \Sigma$. However, by inductive hypothesis $\mathfrak{M} \vDash \phi$. Therefore, by the notion truth in model 3.2, $\mathfrak{M} \vDash \neg \neg \phi$.

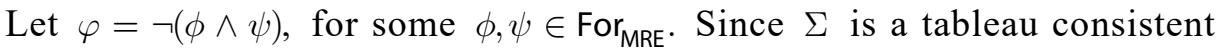
closure under TR, by application of tableau rule $(\neg \wedge), \neg \phi \in \Sigma$ or $\neg \psi \in \Sigma$. .However, by inductive hypothesis, $\mathfrak{M} \vDash \neg \phi$ or $\mathfrak{M} \vDash \neg \psi$. Therefore, by the notion of truth in model 3.2, $\mathfrak{M} \vDash \neg(\phi \wedge \psi)$.

For the remaining cases, the proofs are analogous, since we have at disposal the corresponding tableau rules.

Theorem 4.11 (Completeness Theorem) Let $\Sigma \cup\{A\} \subseteq$ For $_{M R E}$. Then $\Sigma \vDash A \Rightarrow \Sigma \triangleright_{T R} A$.

Proof. Let $\Sigma \cup\{A\} \subseteq$ For $_{\text {MRE. }}$. Suppose it is not the case that $\Sigma \triangleright_{T R} A$. So by the definition 4.4 , for any finite $\Sigma^{\prime} \subseteq \Sigma$ there is a tableau consistent closure $\Delta^{\prime}$ of $\Sigma^{\prime} \cup\{\neg A\}$ under TR such that $\Sigma^{\prime} \cup\{\neg A\} \subseteq \Delta^{\prime}$. Therefore exists a tableau consistent closure $\Delta$ of $\Sigma \cup\{\neg A\}$ under $T R$ such that $\Sigma \cup\{\neg A\} \subseteq \Delta$. Otherwise, any of such closure under $T R$ would be tableau inconsistent. But by the definition of tableau closure 4.3, this would mean that for some finite $\Sigma^{\prime} \subseteq \Sigma$ no closure of $\Sigma \cup\{\neg A\}$ under $T R$ is tableau consistent. As a consequence, by the lemma 4.10, there is a model $\mathfrak{M}$ for MRE such that $\mathfrak{M} \vDash \Sigma \cup\{\neg A\}$. Therefore $\Sigma \not \models A$.

Therefore having theorems 4.7 and 4.11 , we can put them together and obtain the final theorem.

Theorem 4.12 (Adequacy Theorem) Let $\Sigma \cup\{A\} \subseteq$ For $_{\text {MRE }}$. Then $\Sigma \vDash_{\text {MRE }}$ $A \Leftrightarrow \Sigma \triangleright_{T R} A$.

\section{EXPRESSION POWER OF MRE}

An intriguing question appears: what new does MRE bring to the field of positional logic? In particular, are there any essentially new thesis of MRE in comparison to MR? 
To answer the last question, let us define a special substitution function $\sigma:$ For $_{\mathrm{MRE}} \rightarrow$ For $_{\mathrm{MR}}:$

1. $\sigma(A)=A$, if $A \in$ For $_{\mathrm{MR}}$,

2. $\sigma(A * B)=\sigma(A) * \sigma(B)$,

3. $\sigma(\neg A)=\neg \sigma(A)$,

4. $\sigma\left(\mathcal{R}_{\alpha_{1}, \ldots, \alpha_{n}}(A)\right)=\mathcal{R}_{\alpha_{i}}(A)$, for $\alpha_{1}, \ldots, \alpha_{n} \in \mathrm{PL}, A \in \mathrm{AE}_{\mathrm{MRE}}$ and for some $1 \leq i \leq n$

5. $\sigma\left(\vartheta_{i}^{n}\left(\alpha_{1}, \ldots, \alpha_{n}\right)\right)=\mathcal{R}_{\alpha_{k}}(A)$, for all $\vartheta_{i}^{n} \in \mathrm{PS}, \alpha_{1}, \ldots, \alpha_{i} \in \mathrm{PL}$, and for some $1 \leq k \leq n, A \in \mathrm{AE}_{\mathrm{MRE}}$.

As we see any function $\sigma$ : is an identical function for formulas from For $_{\text {MR }}$ (1.), is neutral with respect to the classical connectives $(2 ., 3$.), and reduces occurrences of positional letters in $\mathcal{R}$-formula to the first occurring letter (4.). It is additionally obvious (by 5.) that $\sigma\left(\vartheta_{i}^{n}\left(\alpha_{1}, \ldots, \alpha_{n}\right)\right) \in$ For $_{\text {MR }}$, for all $\vartheta_{i}^{n} \in \mathrm{PS}$ and $\alpha_{1}, \ldots, \alpha_{n} \in \mathrm{PL}$. Clearly, also for all $\sigma, \sigma\left(\right.$ For $\left._{\mathrm{MRE}}\right)=$ For $_{\mathrm{MR}}$. Now, we can form a theorem:

Theorem 5.1 Let $\phi \in$ For $_{\text {MRE. }}$. Then $\vDash_{M R E} \phi$ iff:

- if $\phi \in$ For $_{M R}$ then $\vDash_{M R} \phi$,

- if $\phi \notin$ For $_{M R}$ then for all $\sigma, \vDash_{M R} \sigma(\phi)$.

Proof. The proof 'from right to left' is obvious, since $\sigma(\phi) \in$ For $_{\mathrm{MR}}$. Then if a formula is valid in MR then it can be be proved in the tableaux for MR, and so in the tableaux for MRE (the difference between both sets of tableau rules lies only in the multiple occurrences of positional letters: tableaux for MR are special instances of tableaux for MRE). Therefore by adequacy theorem 4.12 the formula is valid in MRE.

On the other hand, for the opposite implication we repeat the same manoeuvre, observing that any tableau proof in MRE can be rewritten with replacing expressions by $\sigma$ and the result is a correct tableau proof in MR.

Then as a conclusion we have: MRE is a conservative-like extension of MR (see an analogous issue for a quantifier extension in JARMUŻEK and Pietruszczak 2004, 160-161, and Rasiowa and Sikorski 1968). So, there is no any new thesis in MRE in comparison to MR. However, MRE allows you to create theories that you will not create in pure MR, since this logic is a language extension. Below are some examples. 


\section{EXAMPLES OF APPLICATIONS}

As the language of MRE enables to express the state of affairs in more details than MR, its application can be broader. It can be used in cases when the context is more complex - more factors should be taken into account and relations between them are crucial.

One of those applications were pointed out it Malinowski, Pietrowicz, and Szalacha-Jarmużek (2020), where authors considered to use similar extensions of MR to describe social phenomena. According to the authors, sequence of positions $\Gamma=\left(a_{1}, . ., a_{10}\right)$ could be interpreted as for example (ibid., 252):

- $a_{1}$-institution,

- $a_{2}$ - organization,

- $a_{3}$ - social group,

- $a_{4}$-place,

- $a_{5}$-time,

- $a_{6}$ - position in a group,

- $a_{7}$ - social role,

- $a_{8}$-interaction,

- $a_{9}$-individual,

- $a_{10}$-culture.

According to this interpretation, the expression $\mathcal{R}_{\Gamma}(p)$ would mean that phenomenon denoted by the sentence $p$ has happened in the context $\Gamma$.

In MRE we are able to say more than that. We are able to state some relation between the positions. Let us say that: some organization $\mathrm{X}$ (denoted by $a_{2}$ ) organizes a protest (a fact of organizing the protest is denoted by $p$ ), in some big city $\mathrm{Y}$ (denoted as $a_{4}$ ), in some time $\mathrm{H}$ (denoted as $a_{5}$ ). Then let us say that $P$ denote relation of being a part of an organization. Let $a_{9}$ denote any individual. Then:

$$
\mathcal{R}_{a_{2}, a_{4}, a_{5}}(p) \wedge P\left(a_{9}, a_{2}\right) \rightarrow \mathcal{R}_{a_{4}, a_{5}, a_{9}}(p)
$$

So we can express some simple kind of the phenomenon of Peer Pressure. This is of course oversimplification, without going into sociological details.

The other example of application of MRE comes from the metamathematics. We can use the extended positional logic to express axioms 
dependencies or even relation of a system constructed with one set of axioms to the others.

Let $a_{1}, a_{2}, a_{3}, a_{4}$ denote four different theorems and $p$ denote the fact that they have some property, for example that they are consistent. In turn let $P$ denote that the fourth its argument is a logical consequence of the first three arguments in some logic. Then we have:

$$
\neg \mathcal{R}_{a_{1}, a_{4}}(p) \wedge P\left(a_{1}, a_{2}, a_{3}, a_{4}\right) \rightarrow \neg \mathcal{R}_{a_{1}, a_{2}, a_{3}}(p)
$$

So, this expression says that if $a_{1}$ and $a_{4}$ are inconsistent and from $a_{1}$, $a_{2}, a_{3}$ follows $a_{4}$, then also $a_{1}, a_{2}, a_{3}$ are inconsistent.

Using MRE we can present an example of application to the field of mathematics by describing points in the three-dimensional Euclidean space. Let our space be $\mathbb{R}^{3}=\{(x, y, z): x, y, z \in \mathbb{R}\}$. Let $P$ denote a relation between two points that at least one coordinate is equal to the coordinate on the same place of the second point. Let $p$ denote a sentence "x-coordinate of a point equals to 1." Let $a_{1}, \ldots, a_{6}$ denote some entities from the set $\mathbb{R}$ Then, the expression:

$$
\mathcal{R}_{a_{1}, a_{2}, a_{3}}(p) \wedge \mathcal{R}_{a_{4}, a_{5}, a_{6}}(p) \rightarrow P\left(a_{1}, a_{2}, a_{3}, a_{4}, a_{5}, a_{6}\right)
$$

says that if we have two points $\mathrm{x}_{1}, \mathrm{x}_{2} \in \mathbb{R}^{3}$ and for both it is true that the $x$ cooridnate is equal to 1 , then it is also true that $P\left(\mathrm{x}_{1}, \mathrm{x}_{2}\right)$.

Given examples shows that the possible usage of MRE seems wide enough to be present in metalogic, mathematics and social studies. The expression power of a language which does not only contain $\mathcal{R}$-operator, but also possibility of describing the context in which we want to distinguish more than one factor and show some relations between them, can largely influence variety of applications of positional logics. Examples taken from the social studies could be easily transferred onto less complex natural sciences. Whereas example of a usage within the field of formal sciences can lead to grasping the existing theorems and laws in a new manner and can be applied to study relations between formal systems in general.

\section{ACKNOWLEDGMENT}

The research of Tomasz Jarmużek presented in this article was financed by the National Science Centre, Poland, grant No.: UMO-2015/19/B/HS1/02478. 


\section{BIBLIOGRAPHY}

Hıż, Henryk. 1951. Review: "Jerzy Los. Foundations of the Methodological Analysis of Mill's Canons." Journal of Symbolic Logic 16, issue 1: 58-59.

JarmuŻeK, Tomasz, and Andrzej Pietruszczak. 2004. "Completeness of Minimal Positional Calculus." Logic and Logical Philosophy 13: 147-162. doi: 10.12775/ LLP.2018.013.

JarmuŻeK, Tomasz, and Marcin TKaczyk. 2015. Normalne logiki pozycyjne [Normal Positional Logics]. Lublin: Towarzystwo Naukowe KUL.

JARMuŻeK, Tomasz, and Marcin TкасZYк. 2019. "Jerzy Łoś Positional Calculus and the Origin of Temporal Logic." Logic and Logical Philosophy 28: 259-276. doi: 10.12775/LLP. 2018.013

JARMUŻeK, Tomasz. 2013. Jutrzejsza bitwa morska. Rozumowanie Diodora Kronosa [Tomorrow Sea-Fight: Diodorus Cronus' Argument]. Toruń, Wydawnictwo Naukowe UMK.

JARMUŻEK, Tomasz. 2007. "Minimal Logical Systems with $\mathcal{R}$-operator: Their Metalogical Properties and Ways of Extensions." In Perspectives on Universal Logic, edited by JeanYves Béziau and Alexandre Costa-Leite, 319-33. Polimetrica International Scientific Publisher, Italy.

JARMuŻeK, Tomasz. 2018. On the Sea Battle Tomorrow That May Not Happen: A Logical and Philosophical Analysis of the Master Argument. Berlin, Warsaw: Peter Lang Publishing.

JARMUŻeK, Tomasz. 2006. "Rekonstrukcje Rozumowania Diodora Kronosa w ontologii czasu punktowego [Reconstruction of Diodorus Cronus' Argument in Frame of Ontology of Time Consisted of Points].” Analiza i Egzystencja 3: 197-215.

JARMuŻeK, Tomasz, and Mateusz Klonowski. 2020. "Some Intensional Logics Defined by Relating Semantics and Tableau Systems." In Logic in High Definition. Trends in Logical Semantics, edited by Alessandro Giordani and Jacek Malinowski, 33-51. Wien, New York: Springer Verlag

Łoś, Jerzy. 1948. "Logiki wielowartościowe a formalizacja funkcji intensjonalnych [Multivalued Logics and Formalization of Intensional Functions]." Kwartalnik Filozoficzny 17, No. 12: $59-78$.

Łoś, Jerzy. 1947. "Podstawy analizy metodologicznej kanonów Milla [Foundations of Methodological Analysis of Mill's Canons]." Annales Universitatis Mariae Curie-Skłodowska 2.5. F: 269-301.

KarcZewsKa, Anna Maria. 2018. "Maximality of the Minimal $\mathcal{R}$-logic." Logic and Logical Philosophy 27: 193-203. doi: 10.12775/LLP.2017.008

LeCHNIAK, Marek. 1988. "Logika epistemiczna Jerzego Łosia a teoria racjonalnego zachowania [Epistemic Logic of Jerzy Łoś and the Theory of Rational Behaviour].” Roczniki Filozoficzne 26, issue 1: 79-91.

Malinowski, Jacek, Krzysztof Pietrowicz, and Joanna Szalacha-JarmużeK. 2020. "Logic of Social Ontology and Łoś Operator." Logic and Logical Philosophy 29: 239-258. doi: 10.12775/LLP.2020.005.

Rasiowa, Helena, and Roman SiKonski. 1968. Mathematics of Metamathematics. $2^{\text {nd }}$ editon. Warszawa: PWN.

Rescher, Nicholas, and Alasdair URquhart. 1971. Temporal Logic, Library of Exact Philosophy. Wien, New York: Springer Verlag.

Garson, James, and Nocholas Rescher. 1968. "Topological Logic.” Journal of Symbolic Logic 33 , issue 4: $537-48$. 
Suszko, Roman. 1949. Review: "Jerzy Los, Many-Valued Logics and Formalization of Intensional Functions." Journal of Symbolic Logic 14: 64-65.

TKACZyK, Marcin. 2009. Logika czasu empirycznego [Logic of Physical Time]. Lublin: Wydawnictwo KUL.

TKACZYK, Marcin. 2013. "Distribution Laws in Weak Positional Logics.” Roczniki Filozoficzne 66, nr 3: 163-179. doi: 10.18290/rf.2018.66.3-8.

Ткасұук, Marcin. 2013. "Negation in Weak Positional Calculi." Logic and Logical Philosophy 22: 3-19. doi: 10.12775/LLP.2013.001.

\section{ON SOME LANGUAGE EXTENSION OF LOGIC MR: A SEMANTIC AND TABLEAU APPROACH}

\section{S u m m a ry}

In the article we present an extension of the minimal, normal positional logic, i.e., the logic with realization operator MR. Positional logic is a philosophical logic that makes it possible to relate sentences to contexts that can be understood in many ways. We enrich the basic language of minimal positional logic with additional expressions built with predicates and positional constants. We also accept expressions built with the realization operator and many positions, like: $\mathcal{R}_{a_{1}, \ldots, a}(A)$.

Thanks to this, we increased the expressivity of minimal positional logic. In the article we point to many examples of the fact that, thanks to this small change, complex theories based on the proposed extension can be created. As a theory of proof for our logic, we assume tableau methods, showing soundness and completeness theorems. At the end, however, we show that the logic studied here is only a language extension of the MR: all theorems of the extension have their equivalents in pure MR theorems. However, theories built upon the proposed extension can express much more than theories built upon pure MR.

Keywords: extension of minimal positional logic; MR; positional logic; realization operator; tableau methods.

\section{O PEWNYM JEZZYKOWYM ROZSZERZENIU LOGIKI MR: PODEJŚCIE SEMANTYCZNE I TABELAU}

\section{Streszczenie}

W artykule przedstawiamy rozszerzenie minimalnej, normalnej logiki pozycyjnej, czyli logiki z operatorem realizacji. Logika pozycyjna to logika filozoficzna, która umożliwia odniesienie zdań do kontekstów, które można rozumieć na wiele sposobów. Wzbogacamy podstawowy język minimalnej logiki pozycyjnej o dodatkowe wyrażenia zbudowane z predykatów i stałych pozycyjnych. Akceptujemy również wyrażenia zbudowane $\mathrm{z}$ operatorem realizacji oraz wiele pozycji, takich jak: $\mathcal{R}_{a_{1}, \ldots, a}(A)$.

Dzięki temu zwiększyliśmy wyrazistość minimalnej logiki pozycyjnej. W artykule wskazujemy na wiele przykładów na to, że dzięki tej niewielkiej zmianie mogą powstać złożone teorie oparte na proponowanym rozszerzeniu. Jako teorię dowodu dla naszej logiki zakładamy metody tableau, pokazujące twierdzenia o poprawności i zupełności. Na koniec jednak pokazujemy, że badana tutaj 
logika jest tylko rozszerzeniem językowym MR: wszystkie twierdzenia o przedłużeniu mają swoje odpowiedniki w czystych twierdzeniach MR. Jednak teorie oparte na proponowanym rozszerzeniu mogą wyrazić znacznie więcej niż teorie oparte na czystej MR.

Słowa kluczowe: rozszerzenie minimalnej logiki pozycyjnej; MR; logika pozycyjna; operator realizacji; metody tableau. 